\title{
Approach to Systemic Mastocytosis: A case series.
}

\author{
Ines Dakhlia ${ }^{1}$, Najah Boussetta ${ }^{2}$, Sinda Dakhlia ${ }^{1}$, Nour Guediche ${ }^{2}$, Sameh Sayhi², and \\ Bassem Louzir ${ }^{3}$ \\ ${ }^{1}$ Hamad Medical Corporation \\ ${ }^{2}$ Military Hospital of Instruction of Tunis \\ ${ }^{3}$ Tunis El Manar University - Tunisia
}

October 14, 2021

\begin{abstract}
Mast cells are found in bone marrow, blood, mucosal, and connective tissues. They migrate into the loose connective tissue of all organs. They play a major role in many physiologic processes however as discussed in this article they can become an aggressive force which can damage the natural biological balance
\end{abstract}

\section{Introduction:}

Mastocytosis is characterized by proliferation and excessive accumulation of mast cells in normal sites of distribution as the result of a hyperplastic response to an abnormal stimulus. $(1,2)$ It is classified into cutaneous mastocytosis $(80 \%)$ and systemic mastocytosis which involves at least one extracutaneous organ (3).We report a case series of patients presenting with vague cutaneous and gastrointestinal symptoms which were diagnosed with indolent systemic mastocytosis after intensive investigations. This case series emphasizes on the difficulty of diagnosing indolent systemic mastocytosis (ISM) as its symptoms can resemble intestinal diseases. Therefore, physicians must suspect ISM upon recognizing the combination of its manifestations and confirm the diagnosis with serum tryptase test and histopathological studies. (3) Here we also discuss the classification, clinical presentation, diagnosis, treatment, and prognosis of systemic mastocytosis.

Keywords : Mastocytosis, D816V mutation KIT, Mast cell, Systemic mastocytosis.

\section{Key Clinical message:}

The functions of mast cells include regulation of gastric acid secretion, regulation of the microvasculature, and repair and remodeling of connective tissues(1).However when these factors are deranged this can lead to mastocytosis. We present 2 cases of elderly women in their 60 s who were admitted for exploration of unexplained systemic symptoms that were extensively investigated over years with several relapses and symptom free periods. Following an exhaustive search, the diagnosis of mastocytosis was retained and the patients were started on the appropriate treatments with net improvement. Our aim is to raise awareness and try to reduce the typical delay in diagnosis.

\section{Case presentation:}

Case 1.

We report a 66 year old lady known case of hypertension and dyslipidemia who has been hospitalized numerous times since 2014 for recurrent unexplained diarrheal episodes, syncope, palpitations and flushing associated with maculopapular rash in the upper limbs and v-line explored with a punch biopsy of the cutaneous lesions, Esophagogastroduodenoscopy (OGD) and colonoscopy that were unremarkable. The patient was last admitted in our department for severe hypotension not associated with any particular 
triggers. Physical examination showed innumerable $2-4 \mathrm{~mm}$ thin pink papules scattered along the neck and extremities that would urticate after scratching (positive Darier sign) associated with dermographism. The possible etiologies were worked up, including; Pheochromocytoma yielding normal serum metanephrine and catecholamines; Peptide hormones found in Gastrinoma, VIPoma and Somatostatinoma were within normal range. Hyperaldosteronism was excluded with normal serum renin to aldosterone ratio. Relevant imaging with abdominal US, chest $\mathrm{x}$ ray and MRI brain were normal.

In light of complaints of severe chronic pruritus along with the generalized flushing episodes, we suspected the presence of a mast cell disease, thus, further investigation for mastocytosis was initiated. This showed normal levels of chromogranin A, IgE levels and skin prick test. The diagnosis of Mastocytosis was retained upon the presence of 1 major and 2 minor criteria according to WHO 2016 classification; Bone marrow biopsy showing infiltration with many abnormal mast cells with coarse cytoplasmic granules, Immunohistochemistry with anti CD117 antibodies showing large aggregates of mast cells and elevated serum tryptase levels $(27.1 \mathrm{ug} / \mathrm{L})$ respectively. (4) However CKIT mutation at codon 816 analysis was absent. The patient was initiated on anti-leucotriene (singular) and histamine $\mathrm{H} 2$ receptor antagonist (famodine) and Tyrosine kinase inhibitor (Glivec) and was advised to avoid specific triggers such as: Non steroidal anti-inflammatory drugs, opiates and contrast as well as products containing histamine like alcohol, strawberries and tomatoes. Avoidance of extremes of temperature was advised. She was also counselled on auto-injection with adrenaline if she develops anaphylactic symptoms. With strict adherence to medication and lifestyle changes, the patient's symptoms improved and did not have any relapses to this date.

\section{Case 2.}

We report a 65 year old lady with no significant past medical history who presented with 17 months history of $20 \mathrm{~kg}$ weight loss associated with vomiting, diarrhea ,abdominal pain and bloating not associated with any particular triggers for which she consulted a private gastroenterologist on 2 occasions. She underwent a series of examination; an abdominal ultrasound, OGD and colonoscopy with biopsy that showed collagenous colitis, hiatal hernia. Gastric biopsy showed chronic gastritis due to $\mathrm{H}$ pylori and patient was put on symptomatic treatment with no improvement. The patient was then admitted for Intractable vomiting. A thorough physical examination was unremarkable. Laboratory findings showed high vitamin B12 levels with normocytic anemia and hyperglobulinemia. The suspicion of hematological malignancy lead to further investigation with tumor markers (ACE, CA19-9, CA135) which were normal.A CT abdomen showed an enlargement of the pancreas in the cephalic portion and peri-pancreatic and peritoneal infiltrates as well as moderate ascites along with multiple celio-mesenteric lymph nodes. Bone marrow biopsy showing medullary infiltrates of $60 \%$ mastocytes in conjunction with systemic mastocytosis without associated hematological problem and elevated serum tryptase levels at $22.4 \mathrm{ug} / \mathrm{L}$ (4). Molecular analysis on a bone marrow aspirate did not detect a c-Kit D816V point mutation at codon 816 was not present. Based on the patient's pathology and gastrointestinal symptoms, she met the WHO criteria for systemic mastocytosis. This diagnosis was retained upon the presence of 1 major and 1 minor criteria according to WHO 2016 classification. The patient was counselled on avoidance of specific triggers and immediately started on Anti H1, anti H2(famodine and deslor) and anti-leucotrienne (Montelukast). Despite being adherent to medications, the patient developed erythematous cutaneous lesions on her thighs making this an aggressive form of systemic mastocytosis; hence we escalated the treatment with INF alpha 2 blocker, systemic corticosteroids, cladribine and Imatinib.

\section{Discussion:}

Mastocytosis is a group of clinical disorders that results from tissue mast cell and CD 34/CD117 progenitors proliferation. It is due to clonal disorders, characterized by abnormal growth and/or accumulation of clonal Mast Cells primarily in the skin and bone marrow.(5)CKIT (CD117) is a type 3 tyrosine kinase receptor expressed on mast cells and eosinophils. It plays a critical role in mast cell proliferation and differentiation.(6) $90 \%$ of systemic mastocytosis patients were found to have somatic mutation in the KIT gene due to aspartate to valine substitution in the second catalytic domain.(5) this is due to activation of receptor KIT from exon linked mutation of exon $17 \mathrm{D} 816 \mathrm{~V}$ of the KIT gene. (6) 
The symptoms of mastocytosis arise from the mast cell mediators release into tissues, which cause both local and distant effects when distributed in the circulation.(5) These features can be confounded with digestive diseases such as gluten enteropathy, coeliac disease, irritable bowel disease, endocrinological diseases such as pheochromocytoma and carcinoid syndrome as well as autoimmune diseases such as vasculitis and neurological conditions such as depression and fibromyalgia .(7)Our patients were mainly investigated for digestive and endocrinological diseases. The two most important mediators are histamine and platelet activating factor; Histamine causes vasodilation and constriction of bronchial and gastrointestinal smooth muscles as well as enhances gastric acid production by parietal cells causing diarrhea, angioedema, pruritis and hypotension as seen in the first case. The latter mediator results in similar symptoms in addition to pulmonary edema and arrythmia.

The diagnosis of mastocytosis should be made with complete medical history including triggering factors ,that can cause mast cell degranulation such as emotional stress, physical stimuli (trauma),infections, allergic symptoms (beesting, asthma exacerbation) and drugs such as alcohol, aspirin, NSAIDs, opioids, anticholinergics and radioactive dye (7) Mast cells secrete heparin and chromogranin A in the blood which can be found above normal in Mast cell activation syndrome. This was not the case in our patients who had normal chromogranin A levels. Elevated serum tryptase and histamine are general findings in patients with mastocytosis .(5) Total b-tryptase ratio is $>20$, in the absence of other myeloid disorders, strongly suggests underlying systemic mastocytosis. The absolute level of total tryptase does not predict disease severity but can provide a practical method for assessing the efficacy of therapeutic interventions designed to reduce the mast cell burden. (8)Investigations with biopsies of skin, bone marrow, colon, liver should be reviewed for presence of mast cells if symptoms present within these systems, peripheral blood smear should be done to assess for presence of eosinophilia and white blood cell abnormalities that may suggest a clonal aetiology.(7) A small group of patients with SM present with eosinophilia, which has been reported to be of clinical and prognostic significance.(2) Secondly, the presence of the KIT D816V mutation must be assessed in a sensitive and quantitative manner on Bone Marrow and peripheral blood which was done in our patient and came back negative. (6)

According to the 2016 World Health Organization (WHO) classification system, mastocytosis can be classified into several subtypes: cutaneous, localized and systemic (SM).(4) Clinically, mastocytosis can be indolent or aggressive. Indolent forms are by far the most frequent and include Cutaneous, indolent Systemic (ISM), and smoldering Systemic (SSM). Aggressive Systemic Mastocytosis variants (advanced Systemic), include aggressive Systemic (ASM), Systemic Mastocytosis with an associated hematologic neoplasm (SM-AHN), and Mast Cell leukemia (MCL).(6)90\% of cases of mast cell disease have cutaneous mastocytosis. Types of cutaneous SM include urticarial pigmentosa (UP) diffuse cutaneous mastocytosis (DCM) or solitary mastocytoma of the skin. Dermatographisim as well as Darier's sign may be observed in these patients. (7) Our $1^{\text {st }}$ case had positive dermographism.

By addressing all WHO diagnostic criteria for systemic mastocytosis, including KIT mutational analyses in micro-dissected bone marrow mast cells, S Berezowska et al were able to conclude that almost all adult-onset cutaneous mastocytosis had systemic mastocytosis.(9)

Outside of skin involvement, bone marrow involvement of mast cells is most common. (7) Systemic mastocytosis (SM) occurs when mast cells infiltrate extracutaneous organs such as spleen, liver and bone marrow (5). Systemic mastocytosis with an associated haematological neoplasm SM-AHN tends to be observed in an older population of patients and carries a more aggressive clinical course. Types of associated haematological malignancies include chronic myelomonocytic leukaemia (CMML), myelodysplastic syndrome (MDS), B-cell lymphoma/leukaemia/plasma cell neoplasms, myeloproliferative neoplasms and acute myeloid leukaemia (AML).(7)

Diagnosis of systemic mastocytosis requires according to the WHO criteria at least one major and one minor or at least 3 minor criteria. The major criteria being multifocal infiltrates of mast cells in the bone marrow and other extracutaneous organs (>15 mast cell aggregates) and the minor criteria involves more than $25 \%$ of Bone marrow mast cells or mast cell of extracutaneous organs are spindle shaped, KIT mutation at codon 
816 in extracutaneous organs, Basal serum tryptase $>20 \mathrm{ng} / \mathrm{ml}$ and the presence of CD2 and CD25 Bone marrow mast cell. (5)

In both of our patients the serum tryptase was elevated and the bone marrow was infiltrated with multiple mast cells.

A new study assessing the efficacy of WHO criteria on the diagnosis of mastocytosis has shown that based on the WHO criteria, some patients suffering from a true systemic mastocytosis could not be classified as such.

As specific subtypes of mastocytosis may not fulfill the WHO diagnostic criteria, particularly for the distinction between cutaneous and systemic subtypes, especially among well-differentiated systemic mastocytosis and to a lower extent also for indolent systemic mastocytosis without skin lesions. The distinction between indolent and aggressive systemic mastocytosis requires introduction of further objective criteria to have a more refined diagnosis and classification of mastocytosis, which includes the WHO criteria with the exception of serum tryptase, plus other objective clinical (eg systemic mast cell activation symptoms such as bone marrow dysfunction with cytopenias, organomegaly with organ failure and severe malabsorption with hypoalbuminemia and weight loss and last sized osteolysis with local mast cell infiltration and mastocytosis in the skin) and laboratory data (eg b2-microglobulin and LDH serum levels).(10)

All patients who present with advanced disease should have molecular and cytogenetic testing along with baseline pathological and imaging studies as they carry high risk of progression and transformation, disease related symptomatology including GI, skin, pulmonary and neurological symptoms should also be monitored for increasing frequency. (7)At the present time there is no cure for mastocytosis. Because this disorder can vary greatly in its clinical manifestations, treatment needs to be modified according to the patient's symptoms.

A number of indolent mastocytosis patients have few, if any, symptoms, and therefore require little or no therapy. Solitary mastocytomas usually do not require treatment since these lesions often resolve spontaneously (11).

Management of patients within all categories of mastocytosis includes avoidance of triggering factors such as hymenoptera stings,alcohol and heat (5) All patients with proven clonal Systemic Mastocytosis of any subtype should also be provided with an epinephrine pen to use in case of anaphylaxis and instructed on when to present to the emergency department for evaluation when they develop allergic symptoms. Medical alert bracelets can be considered depending on the severity of the disease. (7) This measure was applied in our cases where the patients were informed to use Epi-pen whenever they felt any allergic symptoms.

Some of the first line agents used in the treatment of mastocytosis include H1 and H2 antihistamines, Systemic and intralesional injection steroids, and aspirin. H1-antihistamines are used for reduction of the skin symptoms such as pruritus, bullae erythema, and flushing, while the $\mathrm{H} 2$ antihistamines are used for the gastrointestinal symptoms including dyspepsia. Steroids are helpful for the mitigating bone pain. The use of systemic steroids is typically limited to specific situations in which mast cell flares must be acutely managed or in the setting of end-organ issues. $(5,7)$ For cutaneous symptoms, intralesional injections of corticosteroids can greatly reduce lesion size and associated symptoms.(11) Acetylic salicylic acid (Aspirin), a non-steroidal anti-inflammatory drug (NSAID) acts by normalizing PGD2 metabolites through irreversible inhibition of cyclooxygenase isoenzymes thus has positive effects in mastocytosis. (5)

Second line agents such as Leucotriene antagonists and cromolyn can be used for pruritus as they help prevent mast cell degranulation. Cromolyn is effective in reducing GI symptoms in addition to bone pain and headaches. (7) Omalizumab(Novartis), a humanized murine monoclonal antibody that binds IgE, with an ability to conjugate in vivo with free serum IgE, thus reduce binding to FCeRI on MCs and basophils (5) it should be considered for use in select patients with severe symptoms that have proven unresponsive to leukotriene antagonists or cromolyn (7).Interferon- $\alpha(\mathrm{IFN}-\alpha)$ and cladribine (2-CdA) are used in patients with aggressive SM it acts through several mechanisms including decreasing Mast Cell mediator release, 


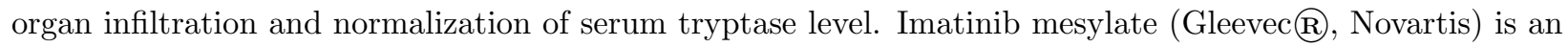
ATP-competitive, orally bioavailable agent and the only FDA-approved inhibitor of various tyrosine kinases for use in patients with Aggressive Systemic Mastocytosis without D816V KIT mutant forms. (5) Both Interferon- $\alpha$ and Imatinib mesylate were used in our patient who relapsed following treatment with first line agents.

A more invasive treatment option centers around a stem cell transplant. Prior to Stem Cell Transplant, combinational therapy should be attempted to bring the patient to best response. With either cladrabine or midostaurin. Transplantation should be considered at the time of best treatment response, particularly for young and otherwise healthy patients with good donor options. (7) Stun et al. investigated the efficacy of allogenic hematopoietic stem cell transplant in patients with advanced Systemic Mastocytosis who underwent sibling/unrelated transplant; this intervention proved to cause a significant decrease in serum tryptase levels and a beneficial effect on long term survival.(5) Despite recommendations of stem cell transplant, no prospective trials have been completed that address key questions regarding transplantation, including optimal timing, debulking strategy, conditioning, or GVHD prophylaxis.(7)

As the phenotypes of systemic mastocytosis are diverse, the prognosis varies from indolent to severe end organ damage and death.(7) Presence of splenomegaly, abdominal lymphadenopathy and ascites are poor prognostic signs. Being an older adult males with anemia, current or previous malignancy, and bone marrow mast cells with lobulated (atypical) mast cell nuclei is also associated with a poorer prognosis. One should monitor the $\mathrm{C}$ finding such as abnormal blood counts and end organ effects as they may suggest evolution of the disease into a more aggressive form.(11)

The overall survival according to the international prognostic scoring system for mastocytosis depends on several risk factors such as male sex, age $>60$, trypstase levels $>12.5 \mathrm{ug} / \mathrm{ml}, \mathrm{Hb}<11 \mathrm{~g} / \mathrm{dl}, \mathrm{ALP}>100 \mathrm{U} / \mathrm{L}$, leukocyte count, platelets $<100 * 109 / \mathrm{L}, \mathrm{LDH}>260 \mathrm{U} / \mathrm{L}$, Calcium $<8.7 \mathrm{mg} / \mathrm{dl}$, Neutrophil $>50 \%$, Skin involvement, Organomegaly, Monocytes $>0.32 \%$, mediator symptoms and allergy. (12)Individuals with low risk disease (cutaneous SM, mastocytosis of skin, indolent SM, smouldering SM) without additional risk factors mentioned above have a70\% or greater overall probability of survival at 24 months. Those in the intermediate risk category with non-advanced disease and presence of one or more risk factors (have a median survival of 13.5 years. Those with advanced disease (regardless of the risk factors) were assigned as high risk with a median survival of 3.5 years.(7)

\section{Conclusion:}

Based on evidence reviewed in discussion section, mastocytosis is a wide group of clonal disorders and can affect many organs including skin, bone marrow and internal organs; as a result it can be mistaken for other gastrointestinal illnesses due to its vague presenting symptoms, it can also be associated with other hematological malignancies and that makes it difficult to diagnose. Physicians should be aware that the main diagnostic investigation is biopsy but one can also check serum tryptase and chromogranin A as done in our cases as well as CKIT mutation analysis.

Treatment of this illness can be challenging as it depends on a case-to-case symptomatology. Our $1^{\text {st }}$ case depicts the successful use of are antihistamines while the $2^{\text {nd }}$ case has shown the efficacy of Interferon alpha and tyrosine kinase inhibitors.

Finally stem cell transplant can be recommended in young adults with a related donor but its timing and debulking method and graft vs host prophylaxis are still not known.

Given the successful recovery achieved in these cases, we suggest that further research such as randomized controlled trials need to be explored in similar cases to harness an approach on investigating mastocytosis.

\section{Authors details with order of contribution:}

\section{1- Dr.Ines Dakhlia, MBBS ${ }^{1}$}

Email: 14006634@rcsi.com 
Contribution: Case identification, manuscript writing and literature review

\section{2- $\quad$ Dr. Sinda Dakhlia, MBBS ${ }^{1}$}

Email: 14006677@rcsi.com

Contribution: Case identification, Manuscript writing and literature review.

\section{3- $\quad$ Dr. Boussetta Najeh MBBS ${ }^{1}$}

Email: boussetta.najah@gmail.com

Contribution: Manuscript writing

\section{4- $\quad$ Dr. Louzir Bassem, MBBS $^{1}$}

Email louzir.bassem@yahoo.fr.

Contribution: Manuscript writing

5- $\quad$ Dr. Nour Gueddiche, MBBS $^{1}$

Email Houdaguediche@gmail.com

Contribution: Manuscript writing

6- Sameh Sayhi, MBBS ${ }^{1}$

Email Sameh_sayhi@yahoo.fr

Contribution: Manuscript writing

Affiliation: Military Hospital Tunisia ${ }^{1}$

Department: Internal Medicine Department.

\section{Acknowledgements:}

I would like to thank Dr. Boussetta Najah, Senior Consultant of Internal Medicine at Military Hospital Tunisia for always supporting young researchers at our institute. I would like to thank the 2 patients for giving us the consent for this manuscript.

\section{Conflict of interest:}

All authors of this manuscript have no conflict of interest to declare.

\section{Ethics statement:}

The patient and her family has given verbal consent to publish this case. The study is conducted ethically in accordance with the World Medical Association Declaration of Helsinki.

\section{Approval from the institutional research body:}

The manuscript is reviewed by the medical research council of Military Hospital Tunis, and has been approved for publication.

\section{Conflict of interest/Disclosure statement:}

The authors certify that they have no conflict of interest and no affiliations with or involvement in any organization or entity with any financial or non-financial interest in the subject matter or materials discussed in this manuscript.

\section{Patient consent:}

Subject has given his verbal informed consent to publish the case. 


\section{Funding Sources:}

NA

\section{Data availability:}

Authors confirm that all relevant data or information are included in the article and is available via open access platform of this journal.

\section{ORCID:}

Ines Dakhliahttps://orcid.org/0000-0002-6301-5037

\section{References:}

1. Laura Ispas MRAH, PhD; and W. James Metzger, MD. The many faces of systemic mastocytosis. ANNALS OF ALLERGY, ASTHMA, \& IMMUNOLOGY VOLUME 87, JULY, 2001. 2001. 2. Ishikawa E ea. Eosinophilia and Bone Lesion as Clinical Manifestations of Aggressive Systemic Mastocytosis. Clin Exp Hematop Vol 53, No 3, Dec 2013. 2013. 3. al. ASa. A rare manifestation of indolent systemic mastocytosis and its management during the coronavirus disease 2019 pandemic; educational lessons from Syria. Annals of Medicine and Surgery 62 (2021) 293-297. 2021. 4. Peter Valent CA, 2 and Dean D. Metcalfe3. Mastocytosis: 2016 updated WHO classification and novel emerging treatment concepts. BLOOD, 16 MARCH $2017 \mathrm{x}$ VOLUME 129, NUMBER 11. 2017;129. 5. Daniel Elieh Ali Komi1 TRSWh, 5. Mastocytosis: from a Molecular Point of View. Clinic Rev Allerg Immunol (2018) 54:397-411. 2018. 6. al Te. Mastocytosis. Am J Clin Pathol 2021;155:239-266 DOI: 101093/ajcp/aqaa183. 2021. 7. Robyn M. Scherber1 aUB. How we diagnose and treat systemic mastocytosis in adults. British Journal of Haematology, 2018, 180, 11-23. 2018. 8. Schwartz LB. Clinical utility of tryptase levels in systemic mastocytosis and associated hematologic disorders. Leukemia Research 25 (2001) 553-562. 2001. 9. al SBe. Adult-onset mastocytosis in the skin is highly suggestive of systemic mastocytosis. Modern Pathology (2014) 27, 19-29. 2014. 10. al LSn-Moe. Evaluation of the WHO criteria for the classification of patients with mastocytosis. Modern Pathology (2011) 24, 1157-1168. 2011. 11. Joseph L. Jorizzo MPaC. Mastocytosis. Current Problems in Dermatology September-October 1998 Volume 10, Issue 5Pages 177-210 pdf. 1998. 12. al. Se. International prognostic scoring system for mastocytosis (IPSM): a retrospective cohort study. Lancet Haematol 2019 December 01; 6(12): e638-e649 doi:101016/S2352-3026(19)30166-8. 2019. 